\title{
Low Temperature Magnetic Properties of Ferromagnetic Layered Composites
}

\begin{abstract}
A. URBANIAK-KUCHARCZYK*
Faculty of Physics and Applied Informatics, University of Łódź, Pomorska 149/153, 90236 Łódź, Poland

A ferromagnetic layered composite ABAB....ABA is studied by means of Green function approach. Our attention is focused on the elementary excitations and low temperature magnetisation behavior. In the presented paper we take also into account the effects of damping due spin-spin interaction. As a result not only the positions and relative intensities of resonance peaks are obtained, but also the resonance line-width is found. On the basis of Green function method the temperature dependence of magnetization of a ferromagnetic layered composite is calculated and the spin wave parameter $B$ in the Bloch's law $T^{3 / 2}$ is found and presented in dependence of parameters characterizing the system under consideration.
\end{abstract}

DOI: 10.12693 /APhysPolA.126.208

PACS: 75.70.-i, 75.70.Cn, 75.30.Ds

\section{Introduction}

In the last two decades properties of periodic composite magnetic materials like photonic [1], phononic [2] or magnonic [3], generated a great deal of interest because of growing technological importance, in view of applications of such materials for information storage, magnetic heads and propagation elements, as well as developments in experimental techniques. Theoretical methods intensively used in investigation of properties of multilayered systems, have been successfully employed to study onedimensional composites [4].

\section{Method and calculations}

We consider a ferromagnetic layered composite $\mathrm{ABAB}$....ABA, where $\mathrm{A}$ and $\mathrm{B}$ are different homogeneous ferromagnetic materials and each block $\mathrm{A}$ is made of $N_{A}$ layers and each block B is made of $N_{B}$ layers. The system is described by Heisenberg Hamiltonian consisting of the exchange, single ion anisotropy, Zeeman and dipolar coupling terms. We denote by $A_{1}$ and $A_{2}$ the exchange integrals for sublayers $\mathrm{A}$ and $\mathrm{B}$, respectively, while $A_{12}$ stands for the parameter of exchange interaction between spins belonging to interface layers in different magnetic sublayers.

In order to calculate magnetic properties of the system it is convenient to apply the Green function method in Random Phase Approximation (RPA) [5, 6]. Magnetisation of the layer $\nu$ in the multilayer system consisting of $p$ of $\mathrm{AB}$ blocks is given by:

$$
\begin{aligned}
& \left\langle S_{\nu}^{z}\right\rangle=S-\phi_{\nu} ; \phi_{\nu}= \\
& \frac{1}{n} \sum_{\vec{h}} \sum_{i=1}^{N+M} \frac{b_{\nu}^{2}\left(k_{i}\right)}{e^{\left(\frac{E\left(k_{i}, h_{x}, h_{y}\right)}{k_{B} T}\right)}-1},
\end{aligned}
$$

where $b_{\nu}\left(k_{i}\right)$ stand for amplitudes of spin waves with

*e-mail: aurbaniak@uni.lodz.pl wave vectors $k_{i}$ and energy $E\left(k_{i}, h_{x}, h_{y}\right)$, propagating in the system. A set of equations for coefficients $b_{\nu}\left(k_{i}\right)$ has been obtained in the following form:

$$
\begin{aligned}
& {\left[\frac{D_{S 1}}{A_{1}}-\alpha\left(k_{i}\right)\right] b_{1}\left(k_{i}\right)+b_{2}\left(k_{i}\right)=0,} \\
& b_{v-1}\left(k_{i}\right)-\alpha\left(k_{i}\right) b_{\nu}\left(k_{i}\right)+b_{v+1}\left(k_{i}\right)=0, \\
& b_{N_{A}-1}\left(k_{i}\right)-\left[\frac{D_{I}}{A_{1}}-\alpha\left(k_{i}\right)\right] b_{N_{A}}\left(k_{i}\right) \\
& +\frac{A_{12}}{A_{1}} b_{N_{A}+1}\left(k_{i}\right)=0 \text {, } \\
& \frac{A_{12}}{A_{2}} b_{N_{A}-1}\left(k_{i}\right)-\left[\frac{D_{I}}{A_{2}}-\alpha\left(k_{i}\right)\right] b_{N_{A}+1}\left(k_{i}\right) \\
& +b_{N_{A}+2}\left(k_{i}\right)=0 \text {, } \\
& b_{N_{A}+v-1}\left(k_{i}\right)-\alpha\left(k_{i}\right) b_{N+v}\left(k_{i}\right)+b_{N_{A}+v+1}\left(k_{i}\right)=0 \text {, } \\
& b_{p\left(N_{A}+N_{B}\right)-1}\left(k_{i}\right) \\
& -\left[\frac{D_{S 2}}{A_{2}}-\alpha\left(k_{i}\right)\right] b_{p\left(N_{A}+N_{B}\right)}\left(k_{i}\right)=0 .
\end{aligned}
$$

In Equation (2) $D_{I}$ is the anisotropy at the surface between $\mathrm{A}$ and $\mathrm{B}$ sublayers and $D_{S 1}$ and $D_{S 2}$ denote surface anisotropy at the surface belonging to the block A and B, respectively. The term $\alpha\left(k_{i}\right)=2 \cos \left(k_{i}\right)$ is proportional to the energy of elementary excitation [5]. The set of allowed values of $k_{i}$ can be found by solving the system of equations (2), employing transfer matrix method [4] and then, the magnetisation of the system can be obtained by means of the equation (1). We focus our attention on spin wave parameter $B$ describing decrease of total spontaneous magnetisation with temperature as a result of magnon excitation in low temperature region. This decrease is given by Bloch's law, namely:

$$
M(T)=M(0)\left(1-B T^{3 / 2}\right) .
$$

The increase of spin wave parameter $B$ with decreasing magnetic layer thickness has been discussed in a number of papers, see e.g. [7]. The parameter $B$ has been calculated in dependence of the of the thickness of the system 
and of the filling fraction defined as [4]:

$$
f=\frac{N_{A}}{N_{A}+N_{B}} \text {. }
$$

Results obtained in our calculation are presented in Figs 1, 2.

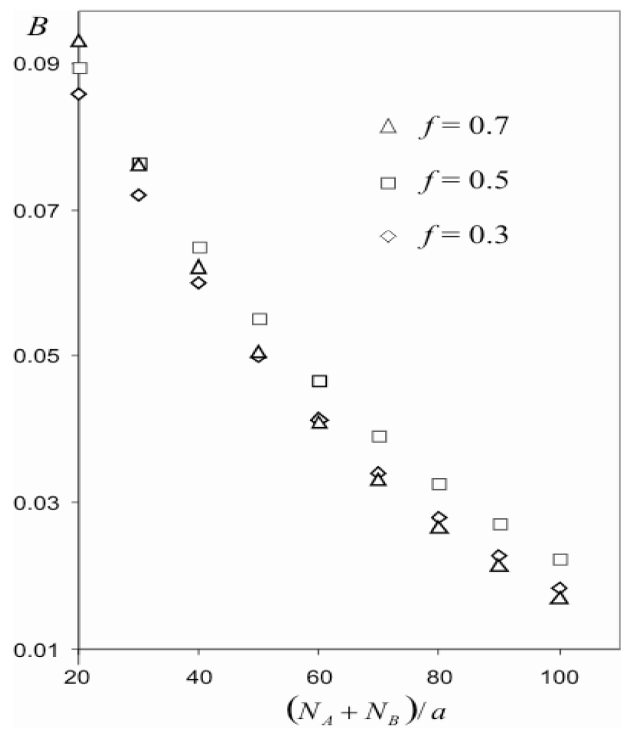

Fig. 1. The dependence of spin wave parameter $B$ on the thickness of block $\mathrm{AB}$ for two filling fractions $f$.

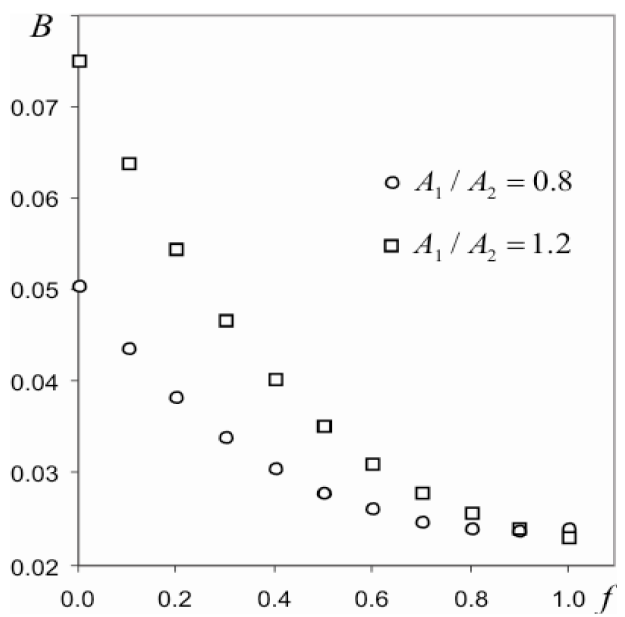

Fig. 2. The dependence of spin wave parameter $B$ on the filling fraction $f$ for two ratios of exchange integrals.

The method applied above can be modified [6] by introducing damping effects due to magnon-magnon interaction [8], calculated on the basis of relaxation equation [9]. As a result a continuous distribution of resonance intensity in Ferromagnetic Resonance (FMR) has been calculated, giving resonance spectra with non-zero line-width. The shape of this spectrum depends on the structure of the multilayer and interaction parameters. Figure 3 gives an example of the dependence of line-width on filling parameter.

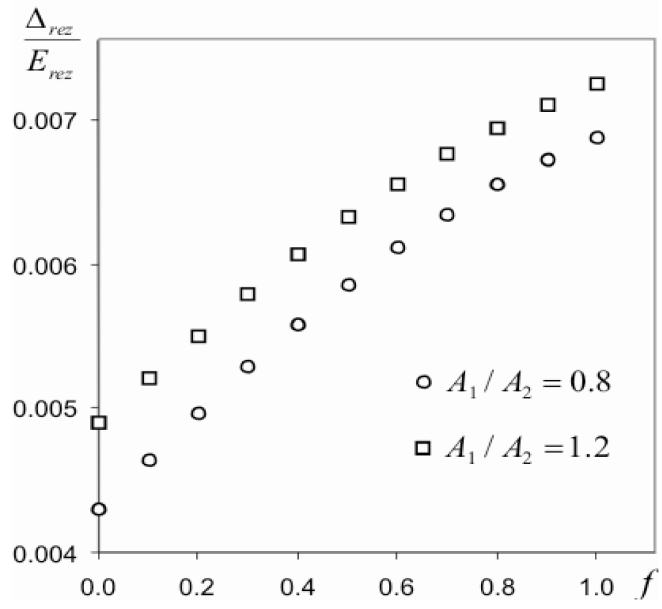

Fig. 3. The dependence of relative line-width $\Delta_{r e z}$ of the first resonance peak (normalized to resonance energy $E_{r e z}$ on the filling fraction for two ratios of exchange integrals.

\section{Conclusions}

The results presented in this paper show an example of modification of the Green function procedure used to study the magnetic properties of magnetic multilayers in such way that it can be employed to study spin wave spectra with non-zero line width and low temperature magnetisation behaviour in one-dimensional composites.

\section{References}

[1] R. Hillebrand, W. Hegert, W. Harms, Phys. Status Solidi 217, 981 (2000).

[2] Y. Tanaka, S. Tamura, Phys. Rev. B 60, 13294 (1999).

[3] M. Krawczyk, H. Puszkarski, Acta Phys. Superficierum 3, 89 (1999).

[4] H. Puszkarski, M. Krawczyk, J.C.S. Levy, D. Mercier, Acta Physica Polonica A 100, 195 (2001).

[5] S. Machowski, A. Urbaniak-Kucharczyk, Surface Science 507-510C, 551 (2002).

[6] A. Urbaniak-Kucharczyk, Acta Phys. Superficierum 13, 162 (2012).

[7] K. Wagner, N. Weber, H.J. Elmers, U. Gradmann, J. Magn. Magn. Mater. 167, 21 (1997).

[8] J.M. Wesselinowa, J. Phys. Condens. Matter 18, 8169 (2006).

[9] L. Wojtczak, Thin magnetic layers, Łódź University Publishing, Łódź 2009, (in Polish). 